\title{
Effect of COVID-19 Pandemic on the Working of Blood Transfusion Center: A Cross-Sectional Study
}

\author{
๑ Ayse Yilmaz, ๑ Haydar Cerullah Zerde** • Berna Alay**, ๑ Zafer Ergul*** \\ Kastamonu Training and Research Hospital, Clinic of Anesthesiology and Reanimation, Kastamonu, Turkey \\ *Kastamonu Training and Research Hospital, Clinic of Blood Transfusion Center, Kastamonu, Turkey \\ **Kastamonu Training and Research Hospital, Clinic of Pediatric Intensive Care Unit, Kastamonu, Turkey \\ ${ }^{* * * K a s t a m o n u}$ University Faculty of Medicine, Department of General Surgery, Kastamonu, Turkey
}

Abstract

\begin{abstract}
Aim: Coronavirus disease-2019 (COVID-19) disease which is unknown how much it will affect the work of hospitals, has caused a pandemic all over world. The decrease in donations due to pandemic required regulation of blood supply in hospitals. In our study, we aimed to evaluate the blood center of our hospital during the pandemic period.

Methods: Patients' records of our hospital blood transfusion center were reviewed retrospectively before and during the pandemic. The patients were divided into two groups as pre-pandemic (11 March-30 June 2019) and pandemic period (11 March-30 June 2020). The groups were compared in terms of age range, gender, blood type, blood type of products, diseases causing transfusion, blood/ blood products transfusion/counts, transfusions performed to COVID-19 patients.

Results: Four thousand two hundred seventy-one blood product transfusions were performed on 1,290 patients. Evaluation of diseases that cause transfusion among statistically significant groups in some diseases such as gastrointestinal diseases, genitourinary system diseases, infectious diseases and other diseases was determined.

Conclusion: While a decrease in our transfusion rates was observed during the pandemic period, the increase in gastrointestinal system diseases is a striking result. The modern world will encounter these and similar pandemics in the future and we think that each region should evaluate its own blood centers in order to prepare for them.
\end{abstract}

Keywords: Blood cells, blood transfusion, pandemics, transfusıon medicine

\section{Introduction}

An outbreak of pneumonia of unknown cause occurred in Wuhan, China, in December 2019. As a result of the investigations, a new coronavirus, Severe acute respiratory syndrome-Coronavirus-2 (SARS-CoV-2) was detected as the cause of this new pneumonia, and this disease was named as Coronavirus disease-2019 (COVID-19) disease (1). It resulted in a pandemic that spread rapidly all over the world. In many viral diseases, both blood donation and blood transfusion procedures can't be applied due to the incubation period, disease-related fever, etc. (2).

The spread of COVID-19 has had a serious impact on blood donation numbers, blood supply and blood safety. The incubation period of SARS-CoV-2 is usually 1-14 days; while the average is 5-6 days, the longest reported is 24 days (3). China, which is considered the starting point of the pandemic, became the first country with a problem in blood transfusion and regulations were made in the country for blood donation (4). Blood center records should be reviewed and blood and blood product sources should be managed as efficiently as possible. The demand must be fully evaluated and a system must be formulated for the emergency supply, demand and use of the blood supply.

With this information, we thought that we should examine the use of blood products in our own hospital. Therefore, our study not only reflects the state of our blood center, but can also guide scientists on what kind of supply and demand might be in other global pandemics. Based on this idea, we aimed to evaluate the blood center

Address for Correspondence: Ayse Yilmaz, Kastamonu Training and Research Hospital, Clinic of Anesthesiology and Reanimation, Kastamonu, Turkey 
of our hospital and analyze how it was affected by the pandemic process.

\section{Methods}

\section{Study Design}

The study was conducted in accordance with the Declaration of Helsinki; Ethics committee approval was obtained from the local Kastamonu Training and Research Hospital Clinical Research Ethics Committee (date: 21/12/2020 and approval number: 2020-KAEK-14311.01). The personal information of the patients was not used, only the data obtained from the blood center records were evaluated. Therefore, permission was obtained from the hospital manager for the examination of records, and there was no need to obtain individual patient consent.

\section{Data Collection}

The present cross-sectional study includes the data of the Blood Transfusion Center of the Kastamonu Traning and Research Hospital and blood and blood product transfusions performed between the pre-pandemic period (11 March-30 June 2019) and the pandemic period (11 March-30 June 2020) were determined. In the study, two different periods were compared, namely the prepandemic period and the pandemic period. For this purpose, data on a total of 1,290 patients, including 747 patients followed up during the pandemic period and 543 patients followed in the pre-pandemic period, were used. Transfusions consist of 4,271 blood product transfusions, including 3234 erythrocytes suspensions (ES), 725 fresh frozen plasma (FFP) and 312 platelet suspensions (TS).

\section{Data Assessment}

The age range, gender, blood type of the patient, the blood type of the transfused product, the diseases that caused the patient's transfusion, and the amount of transfusion performed in the blood center during or before the pandemic were examined. Transfusions for COVID-19 patients have been determined. These patients in the infectious diseases group were taken as COVID-19 definite or doubtful. Patients with a definite diagnosis have positive COVID-19 polymerase chain reaction (PCR) performed by our hospital's laboratory. While the COVID-19 PCR test was negative, there were patients considered suspicious according to lung tomography imaging, and the transfusions applied to these patients were evaluated as transfusions to patients suspected of COVID-19 disease.

\section{Statistical Analysis}

Data obtained from blood center records were encoded and analyzed using SPSS version 22 (IBM). Descriptive analysis was performed to calculate frequencies and ratios. Chi-square test, Kruskal-Wallis test, Fisher's Exact test were used to examine the level of the relationship between variables. The $\mathrm{p}<0.005$ was considered statistically significant.

\section{Results}

Four thousand two hundred seventy-one blood and blood product transfusions were performed on a total of 1,290 patients, including 747 patients treated in the pre-pandemic period and 543 patients followed up during the pandemic period. It was observed that 37 transfusions were made in 17 patients who were positive for COVID-19 during the pandemic period. This consists of 28 ES transfusions for 13 patients, 7 FFPs for 3 patients and 2 TS transfusions for 1 patient. When examining the distribution of the patients in terms of age, gender and blood groups in demographic evaluations; there was a difference in terms of gender $(p=0.034)$ (Table 1$)$.

When the groups of blood and blood products used in the patients were evaluated, there was no difference in shoes ( $p=0.087$ ) between the periods (Graphic 1$)$.

When the use of ES $(p=0.104)$, FFP $(p=0.307)$ and TS $(p=0.232)$ between the periods of the followed-up patients was examined; it was determined that there was no statistically significant difference between the periods for all three (Graphic 2).

While the ratio of female patients, which was $60.8 \%$ in the pre-pandemic period, to $54.9 \%$ during the pandemic period, the rate of male patients increased from $39.2 \%$ to $45.1 \%$.

When the distribution of blood groups is examined, the majority of patients for both periods A Rh $(+)(41.4 \%$ for both periods) and $0 \mathrm{Rh}(+)(28.8 \%$ for the pre-pandemic period and $28.7 \%$ for the pandemic period) blood groups and distributions to other blood groups are also similar.

When the diseases that cause transfusion were compared between periods, there was no statistically significant difference between the two periods in terms of however transfusion due to the musculoskeletal system diseases and trauma $(p=0.03)$ the presence of endocrine, nervous system diseases, cardiovascular system diseases and respiratory diseases ( $p>0.05)$. bleeding-phytic system

\begin{tabular}{|c|c|c|c|c|}
\hline \multirow{2}{*}{ Factor } & \multirow{2}{*}{ Group } & \multicolumn{2}{|l|}{ Period } & \multirow{2}{*}{$p$} \\
\hline & & Pre pandemic & Pandemic & \\
\hline \multirow{4}{*}{ Age range } & $18-30$ & 145 (19.4\%) & $118(21.7 \%)$ & \multirow{4}{*}{0.137} \\
\hline & $31-50$ & 65 (8.7\%) & 58 (10.7\%) & \\
\hline & $51-70$ & 209 (28.0\%) & $134(24.7 \%)$ & \\
\hline & $>70$ & $421(56.4 \%)$ & 336 (61.9\%) & \\
\hline \multirow{2}{*}{ Gender } & Female & $454(60.8 \%)$ & 298 (54.9\%) & \multirow{2}{*}{0.034} \\
\hline & Male & 293 (39.2\%) & $245(45.1 \%)$ & \\
\hline
\end{tabular}




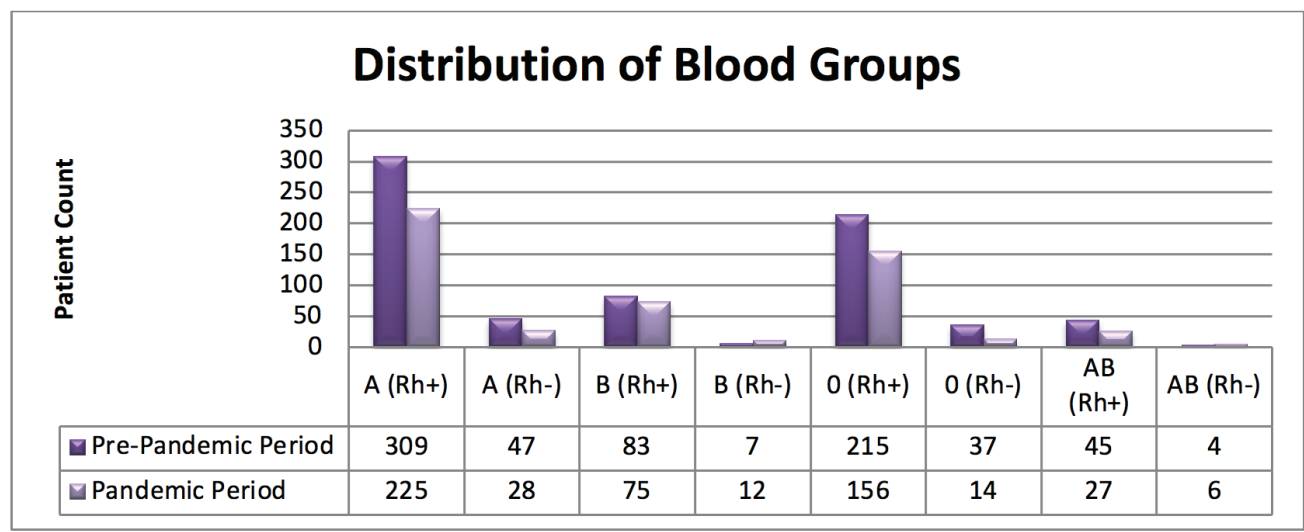

Graphics 1. Distribution of blood groups

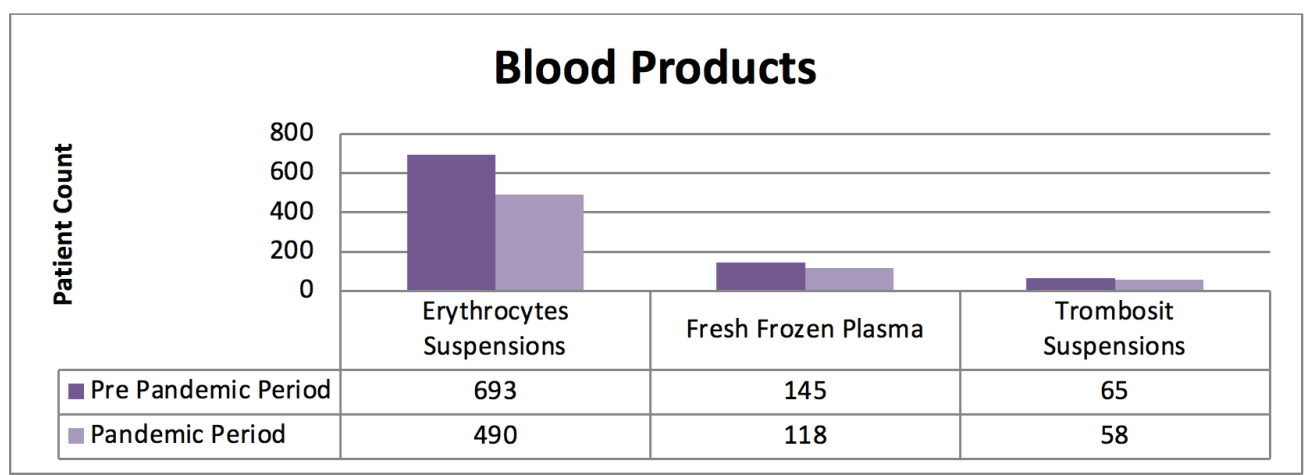

Graphic 2. Distribution of used blood products to patients

It was determined that there is a difference $(p<0.05)$ in terms of diseases, gastrointestinal diseases $(p=0.03)$, genitourinary system diseases $(p<0.01)$, infectious diseases $(p<0.01)$ and other diseases (malignancy, hematology, etc.) $(p<0.01)$ (Table 2).

When the use cases of ES, FFP and TS were examined according to the COVID-19 conditions of 543 patients treated during the pandemic period, there was no statistically significant difference between the groups for ES and FFP ( $p>0.05)$, while for TS there was a significant difference $(p<0.05)$ has been determined. The rate of patients using TS is $8.9 \%$ in the Negative group, $25.5 \%$ in the Suspect group and $4.3 \%$ in the Positive group (Table 3).

When the amount of ES, FFP and TS usage in terms of COVID-19 groups of patients followed up during the pandemic period; It was determined that there was a statistically significant difference between the periods in terms of the amount of ES $(p=0.046)$ use, and no difference for the amount of FFP $(p=0.973)$ and TS $(p=0.362)$ use. The mean of ES use of 430 patients using ES in the Negative group was 2.91 2 2.27, this average was calculated as $3.51 \pm 2.69$ in the Suspect group and $2.15 \pm 2.12$ in the Positive group. The mean use of FFP

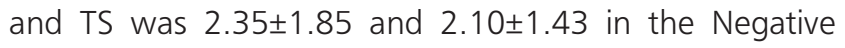

group, $2.21 \pm 1.37$ and $2.64 \pm 2.41$ in the suspect group, respectively and $2.33 \pm 1.53$ and $1.00 \pm 0.0$ in the positive group.

\begin{tabular}{|c|c|c|c|}
\hline \multirow{2}{*}{$\begin{array}{l}\text { Diseases that cause } \\
\text { transfusion }\end{array}$} & \multicolumn{2}{|l|}{ Period } & \multirow{2}{*}{ p } \\
\hline & Pre-pandemic & Pandemic & \\
\hline Endocrine diseases & $13(1.7 \%)$ & $6(1.1 \%)$ & 0.350 \\
\hline Nervous system diseases & $85(11.4 \%)$ & $50(9.2 \%)$ & 0.209 \\
\hline $\begin{array}{l}\text { Musculoskeletal system and } \\
\text { trauma }\end{array}$ & $147(19.7 \%)$ & $73(13.4 \%)$ & 0.003 \\
\hline Cardiovascular diseases & $70(9.4 \%)$ & $55(10.1 \%)$ & 0.650 \\
\hline $\begin{array}{l}\text { Gastrointestinal system } \\
\text { diseases }\end{array}$ & $111(14.9 \%)$ & $115(21.2 \%)$ & 0.003 \\
\hline Respiratory diseases & $111(14.9 \%)$ & $71(13.1 \%)$ & 0.364 \\
\hline $\begin{array}{l}\text { Genitourinary system } \\
\text { diseases }\end{array}$ & $141(18.9 \%)$ & $55(10.1 \%)$ & $<0.001$ \\
\hline $\begin{array}{l}\text { Contagious disease presence } \\
\text { or suspected }\end{array}$ & $1(0.1 \%)$ & $67(12.3 \%)$ & $<0.001$ \\
\hline $\begin{array}{l}\text { Other (malignancy, } \\
\text { hematology etc.) }\end{array}$ & 298 (39.9\%) & $165(30.4 \%)$ & $<0.001$ \\
\hline \multicolumn{4}{|c|}{$\begin{array}{l}\text { Musculoskeletal diseases } \quad(p=0.03) \text {, gastrointestinal diseases } \quad(p=0.03) \text {, } \\
\text { genitourinary system diseases }(p<0.01) \text {. infectious diseases }(p<0.01) \text { and other } \\
\text { diseases (malignancy, hematology. etc.) }(p<0.01) \text { due to a statistically significant } \\
\text { difference was observed between the periods in transfusions, (chi-square test) }\end{array}$} \\
\hline
\end{tabular}




\begin{tabular}{|c|c|c|c|c|}
\hline & COVID-19 & $\mathbf{n}$ & Mean $\pm S D$ & $p$ \\
\hline \multirow{3}{*}{ ES } & Negative & 430 & $2.91 \pm 2.27$ & \multirow{3}{*}{$0.046^{*}$} \\
\hline & Suspect & 47 & $3.51 \pm 2.69$ & \\
\hline & Positive & 13 & $2.15 \pm 2.12$ & \\
\hline \multirow{3}{*}{ FFP } & Negative & 101 & $2.35 \pm 1.85$ & \multirow{3}{*}{0.973} \\
\hline & Suspect & 14 & $2.21 \pm 1.37$ & \\
\hline & Positive & 3 & $2.33 \pm 1.53$ & \\
\hline \multirow{3}{*}{ TS } & Negative & 42 & $2.10 \pm 1.43$ & \multirow{3}{*}{0.362} \\
\hline & Suspect & 14 & $2.64 \pm 2.41$ & \\
\hline & Positive & 2 & $1.00 \pm 0.00$ & \\
\hline
\end{tabular}

When the COVID-19 groups of the patients followed up during the pandemic period were examined in terms of the amount of ES use, it was found that there was a statistically significant difference $(p=0.046)$ between the periods (Kruskal-Wallis test). TS was examined according to the COVID-19 conditions of the patients treated during the pandemic period, there was a significant difference $(p=0.01)$ has been determined (Fisher's Exact test). ES: Erythrocytes suspensions, TS: Trombosit suspensions, FFP: Fresh frozen plasma, COVID-19: Coronavirus disease-2019

\section{Discussion}

In our study, a decrease of $27.3 \%$ was observed in the transfusions applied during the pandemic period compared to the pre-pandemic period. Al-Riyami et al. (5), evaluated the blood centers of 16 countries in the Eastern Mediterranean Region, 75\% of whom were national blood relatives and observed a decrease in their demand in most centers. Our study is compatible with the literature in this respect. In general, while the number of transfusions decreased during the pandemic period, it was observed that there was a relative increase in the need for transfusion in male patients as an interesting result of our study.

In the study of Barriteau et al. (6), 41 (13.4\%) of 305 COVID-19 patients hospitalized were transfused, of which: $33(11.1 \%)$ red blood cells (RBC), 5 (1.6\%) platelets (PLTs) reported that $3(1.0 \%)$ plasma transfusions were performed. In our study, we found that ES transfusion was used the most in COVID-19 patients, and then FFP and TS were used. The proportional distribution of our transfusions among blood products is consistent with this study.

A study examined the distribution of blood groups from community populations and patients with COVID-19 disease, and it was shown that blood group A was associated with an increased risk of infection, while blood type 0 was associated with low risk (7). Our study shows that the blood product that should be supplied the most before and during the pandemic period is the products belonging to the A Rh (+) blood group and it is compatible with the literature. As an interesting result in our study, it was determined that although there are products belonging to the A blood group, which we identify as the most common blood type and which we use the most in general transfusions, in COVID-19 patients, blood products belonging to the 0 blood group are mostly needed.

Another study examined the relationship of $A B 0$ blood groups with COVID-19 disease and evaluated the relationship between the presence of IgG anti-a and this disease as a subgroup. As a result, analyzing the data in this way strongly indicates that the presence of anti-A antibodies, and more specifically IgG anti-A, in serum should be considered as a more important factor than the blood type itself, relative to the relationship with COVID-19. According to this result, there is a correlation between COVID-19 sensitivity and ABO blood types (8). In this context, transfusion process and blood/blood product supply are also very important.

During the COVID-19 pandemic, the selection and preparation of patients who require orthopedic surgery are important and a certain standard has been tried to be established in this regard. In our study, it is observed that the transfusion rates of those performed due to musculoskeletal system diseases during the pandemic period decreased (9). We are of the opinion that the reason for this lowness may be the limitations applied due to the pandemic, the reduction of elective surgeries and the reduction of traumas.

Xiao et al. (10) reported in their study that the SARSCoV-2 virus infects epithelial cells of the stomach, duodenum and rectum. Gu J et al. (11), described COVID-19 disease, increased transaminases, hypoproteinemia, and liver damage caused by prolongation in prothrombin time in their study. In our study, it was observed that transfusions performed due to gastrointestinal diseases increased. The reason for this is that, when epithelitis occurring in the gastrointestinal system and accompanying bleeding disorders come together, it may cause microgastrointestinal hemorrhages in patients. Bleeding of stress ulcers caused by psychological problems during the pandemic period and the use of antiaggregant or anticoagulant drugs during this period may be another reason.

While transfusions originating from the genitourinary system, it regressed during the pandemic period. It was thought that the reason for this situation was the fact that more transfusions were used in elective operations and the number of transfusions naturally decreased when these operations were delayed.

Transfusions in infectious diseases have increased. Hematological changes that occur in COVID-19 disease have been reported to cause normal or slightly decreased hemoglobin and thrombocyte values in most patients $(12,13)$. Here, of course, in most infectious diseases, bleeding disorder is not observed, but in COVID-19 disease, required regulation is affected. In our study, 60 
patients with COVID-19 suspicious and positive patients were treated with ES, 17 patients with FFP and 16 patients with TS transfusion, and naturally, a statistically significant difference was observed between the periods.

Daily routine transfusions are procedures that cover blood losses due to surgery, trauma and oncological reasons. $15 \%$ of erythrocyte transfusions are reserved for hematology and oncology patients (14). In our study, transfusions performed for other reasons decreased during the pandemic period. It is thought that among the reasons for this, routine transfusion patients do not want to come to the hospital due to fear of getting sick, doctors make the decision of transfusion more difficult, reduction of elective surgeries, etc.

In studies conducted, immune plasma therapy appears to be safe, clinically effective in COVID-19 patients, and there are opinions that it reduces mortality $(15,16)$. FFPs included in the study were used in the usual indications in bleeding diathesis, warfarin-related bleeding, prolonged INR, liver failure, as a protein and nutrient source (in albumin deficiency), vitamin K-dependent coagulation factor deficiency, massive transfusion protocol and DIC (17).

Although COVID-19 disease is a viral respiratory disease that can cause the severe acute respiratory syndrome, it may cause susceptibility to thrombotic disease in both venous and arterial circulation, platelet activation disorder, endothelial dysfunction and stasis due to excessive inflammation in patients (18). Chen et al. (19), investigated whether COVID-19 was associated with significant thrombocytopenia in their study, and thrombocytopenia was observed in $12 \%$ of their patients. Investigation of the etiology of thrombocytopenia, clinical history, laboratory values, complete blood count and peripheral smear examination are essential components of the diagnostic study, and physicians should be knowledgeable in the appropriate selection and interpretation of these specific tests. In our study, the rate of platelet use increased from $8.7 \%$ in the pre-pandemic period to $10.7 \%$ in the pandemic period. The difference is not statistically significant, but clinically, this increase, which is concurrent with COVID-19 disease, is significant. We do not yet know how much the COVID-19 pandemic will affect the operation of blood transfusion centers, but the literature should recommend to be prepared $(20,21)$.

\section{Study Limitations}

The limitations of the study are that it is a single center study and the number of transfusions in COVID-19 patients is low because it was conducted at the beginning of the pandemic process. In future studies, evaluation of transfusions made only to COVID-19 patients can give more information to science about the use of blood and blood products in the pandemic. Despite these limitations, we showed in our study that there was a decrease in blood transfusions, an increase in transfusions performed for gastrointestinal reasons and that blood group 0 products were used more in our hospital during the pandemic period.

\section{Conclusion}

Clearly, the decrease in our transfusion rates can be traced in our data results. Another reason for the decrease in the number of transfusions may be that the decision of transfusion becomes more difficult for physicians. In these times when it is not known how long the pandemic will last, more extensive and multi-center studies are needed to use the blood center resources more efficiently. In our study, the increase in transfusion performed in infectious diseases can be considered as a natural result. However, the increase in gastrointestinal system diseases is a striking result. As an interesting result, we found that we mostly use blood products belonging to the 0 blood group in COVID-19 patients, although there are products belonging to the A blood group that we identify as the most common blood group and which we use the most in general transfusions. It is very likely that the modern world will encounter this and similar pandemics in the future periods. We think that each region should evaluate its own blood centers.

\section{Authorship Contributions}

Concept: A.Y., H.C.Z., B.A.., Z.E., Design: A.Y., H.C.Z., B.A.., Z.E., Data Collection or Processing: A.Y., H.C.Z., B.A.., Z.E., Analysis or Interpretation: A.Y., H.C.Z., B.A.., Z.E., Literature Search: A.Y., H.C.Z., B.A.., Z.E., Writing: A.Y., H.C.Z., B.A.., Z.E.

Conflict of Interest: No conflict of interest was declared by the authors.

Financial Disclosure: The authors declared that this study received no financial support.

\section{References}

1. Huang $C$, Wang $Y$, Li $X$, et al. Clinical features of patients infected with 2019 novel coronavirus in Wuhan, China. Lancet 2020;395:497-506.

2. Roberts DJ, Field S, Delaney M, Bates I. Problems and Approaches for Blood Transfusion in the Developing Countries. Hematol Oncol Clin North Am 2016;30:477-95.

3. Chan JF, Yuan S, Kok KH, et al. A familial cluster of pneumonia associated with the 2019 novel coronavirus indicating personto-person transmission: a study of a family cluster. Lancet 2020;395:514-23.

4. Recommendations for blood organizations dealing with the novel coronavirus disease (COVID-19) outbreak (v1.0). China Blood Transfusion Association [Accessed date: 23/03/2021]. 
Available at: http://eng.csbt.org.cn/portal/article/index/ id/606/cid/7.html

5. Al-Riyami AZ, Abdella YE, Badawi MA, et al. The impact of COVID-19 pandemic on blood supplies and transfusion services in Eastern Mediterranean Region. Transfus Clin Biol 2021;28:16-24.

6. Barriteau CM, Bochey P, Lindholm PF, Hartman K, Sumugod $\mathrm{R}$, Ramsey G. Blood transfusion utilization in hospitalized COVID-19 patients. Transfusion 2020;60:1919-23.

7. Li J, Wang $X$, Chen J, Cai $Y$, Deng A, Yang M. Association between $A B O$ blood groups and risk of SARS-COV-2 pneumonia. Br J Haematol 2020;190:24-7.

8. Gérard C, Maggipinto G, Minon JM. COVID-19 and ABO blood group: another viewpoint. Br J Haematol 2020;190:934.

9. Rodrigues-Pinto R, Sousa R, Oliveira A. Preparing to Perform Trauma and Orthopaedic Surgery on Patients with COVID-19. J Bone Joint Surg Am 2020;102:946-50.

10. Xiao F, Tang $M$, Zheng $X$, Liu Y, Li X, Shan H. Evidence for Gastrointestinal Infection of SARS-CoV-2. Gastroenterology 2020;158:1831-3.e3.

11. Gu J, Han B, Wang J. COVID-19: Gastrointestinal Manifestations and Potential Fecal-Oral Transmission. Gastroenterology 2020;158:1518-9.

12. Fan BE, Chong VCL, Chan SSW, et al. Hematologic parameters in patients with COVID-19 infection. Am J Hematol 2020;95:131-4.

13. Sholzberg M, Tang GH, Negri E, et al. Coagulopathy of hospitalised COVID-19: A Pragmatic Randomised Controlled Trial of Therapeutic Anticoagulation versus Standard Care as a Rapid Response to the COVID-19 Pandemic (RAPID COVID COAG - RAPID Trial): A structured summary of a study protocol for a randomised controlled trial. Trials 2021;22:202.

14. Iqbal N, Haider K, Sundaram V, et al. Red blood cell transfusion and outcome in cancer. Transfus Apher Sci 2017;56:287-90.

15. Rajendran K, Krishnasamy N, Rangarajan J, Rathinam J, Natarajan M, Ramachandran A. Convalescent plasma transfusion for the treatment of COVID-19: Systematic review. J Med Virol 2020;92:1475-83.

16. Mandel M, Gurevich M, Mandelboim M, Amital H, Achiron A. Convalescent Whole Blood Donors Screening Strategies for Providing Efficient and Safe COVID-19 Survivors' Plasma and Other Blood Components. Isr Med Assoc J 2021;23:7-10.

17. Farrugia A, Robert P. Plasma protein therapies: current and future perspectives. Best Pract Res Clin Haematol 2006;19:243-58.

18. Carson JL, Grossman BJ, Kleinman S, et al. Red blood cell transfusion: a clinical practice guideline from the AABB* Ann Intern Med 2012;157:49-58.

19. Chen N, Zhou M, Dong $X$, et al. Epidemiological and clinical characteristics of 99 cases of 2019 novel coronavirus pneumonia in Wuhan, China: a descriptive study. Lancet 2020;395:507-13.

20. Kamel $\mathrm{H}$. We stand ready ... Blood collection organizations and the COVID-19 pandemic. Transfusion 2021;61:1345-9.

21. Garcia-Lopez J, Delgadillo J, Vilarrodona A, et al. SARS-CoV-2/ COVID-19 pandemic: first wave, impact, response and lessons learnt in a fully integrated Regional Blood and Tissue Bank. A narrative report. Blood Transfus 2021;19:158-67. 